Direct strain energy harvesting in automobile tires using piezoelectric $\mathrm{PZT}$-polymer composites

This article has been downloaded from IOPscience. Please scroll down to see the full text article.

2012 Smart Mater. Struct. 21015011

(http://iopscience.iop.org/0964-1726/21/1/015011)

View the table of contents for this issue, or go to the journal homepage for more

Download details:

IP Address: 134.221.169.87

The article was downloaded on 20/12/2011 at 07:43

Please note that terms and conditions apply. 


\title{
Direct strain energy harvesting in automobile tires using piezoelectric PZT-polymer composites
}

\author{
D A van den Ende ${ }^{1,2}$, H J van de Wiel ${ }^{1}$, W A Groen ${ }^{1,2}$ and \\ $S$ van der $\mathbf{Z w a a g}^{2}$ \\ ${ }^{1}$ TNO (Dutch National Institute for Applied Scientific Research), Materials Performance Department, \\ Rondom 1, 5612AP, Eindhoven, The Netherlands \\ ${ }^{2}$ Novel Aerospace Materials Group, Faculty of Aerospace Engineering, Delft University of Technology, \\ Kluyverweg 1, 2629 HS Delft, The Netherlands \\ E-mail: daan.vandenende@tno.nl
}

Received 15 July 2011, in final form 27 October 2011

Published 19 December 2011

Online at stacks.iop.org/SMS/21/015011

\begin{abstract}
Direct piezoelectric strain energy harvesting can be used to power wireless autonomous sensors in environments where low frequency, high strains are present, such as in automobile tires during operation. However, these high strains place stringent demands on the materials with respect to mechanical failure or depolarization, especially at elevated temperatures. In this work, three kinds of ceramic-polymer composite piezoelectric materials were evaluated and compared against state-of-the-art piezoelectric materials. The new composites are unstructured and structured composites containing granular lead zirconate titanate (PZT) particles or PZT fibers in a polyurethane matrix. The composites were used to build energy harvesting patches which were attached to a tire and tested under simulated rolling conditions. The energy density of the piezoelectric ceramic-polymer composite materials is initially not as high as that of the reference materials (a macro-fiber composite and a polyvinylidene fluoride polymer). However, the area normalized power output of the composites after temperature and strain cycling is comparable to that of the reference devices because the piezoelectric ceramic-polymer composites did not degrade during operation.
\end{abstract}

(Some figures may appear in colour only in the online journal)

\section{Introduction}

Development of wireless autonomous sensors is a rapidly developing field for monitoring systems ranging from distributed sensing to industrial structural health monitoring [1]. The development of this field depends on the successful marriage of several different disciplines, from ultra-low power data acquisition and processing to efficient energy management. Ultimately in situ energy generation and temporary storage are needed for truly self-sufficient devices [2, 3]. There are many different ways of powering these sensors, such as photovoltaic, thermoelectric and vibration based power sources. The most appropriate converter depends on the power source present in the local environment $[4,5]$.
In certain applications $[6,7]$ direct strain energy harvesting can be an interesting alternative as opposed to harvesting energy using resonant structures, which are considered the current standard in piezoelectric energy harvesting [2, 8-10]. Direct energy harvesting is comparatively useful in environments where low frequency, high strains are present. Direct energy harvesting devices are essentially foil-type devices with limited thickness, which are attached directly to the host structure. The energy harvester will follow the deformations of the host structure, thereby harvesting energy from the environment. Advantages of this type of energy harvesting include higher bandwidth, ease of manufacturing and assembly [11] and ease of structure integration [3]. One of the applications which can benefit from strain 
energy harvesting is the 'smart tire', an automobile tire fitted with sensors, signal conditioning electronics and a data transmitter [12]. It is envisaged that the amount of electronics in automobile tires will increase [12], as will the data rate, therefore increasing power consumption of the tire electronics. This means battery powered modules will not achieve a sufficient lifetime. Several attempts have been made at manufacturing energy harvesters inside automobile tires, using electromagnetic [13, 14] and piezoelectric power generators [9]. However, there are drawbacks associated with all of these types of energy harvesting. The electromagnetic (inductive) systems are relatively bulky [13], generate low voltages [9] or require an external source outside the tire [14], adding to system complexity and cost. Designs based on piezoelectric materials have also been considered many times [9, 15]. Most are based on bender type energy harvesters [9, 17] using ceramic lead zirconate titanate (PZT) in a mass-spring system. This brittle ceramic is not practical to use in automobile tires unless elaborate stress transfer mechanisms are employed [9], requiring very tight manufacturing tolerances in order to function properly. Moreover, PZT ceramics lose their stiffness and piezoelectricity at high strain levels due to mechanical depoling [16, 11], which could affect the output of these types of energy harvesters during their lifetime. Based on the tensile strain limit the piezoelectric polymer PVDF is a more suitable candidate, which provides piezoelectric response up to much higher strains [18]. However, PVDF materials have insufficient thermal properties for use in automobile tires [14], where temperatures may rise to above $80^{\circ} \mathrm{C}$ during operation.

Piezoelectric composites are a class of materials which can be designed to possess both adequate thermal and mechanical properties. For application in automobile tires, a piezoelectric ceramic phase can be embedded inside a high-performance polymer. However, there is always a trade-off between mechanical properties and piezoelectric properties. These properties depend on relative volume fractions of the phases, connectivity type [19] and type of polymer matrix and other reinforcements.

This paper focuses on materials development for direct strain energy harvesting in automobile tires. The piezoelectric ceramic-polymer composite properties can be designed in such a way that they are specifically suited for the application requirements and are well suited to survive the environment of the application.

\section{Theory}

\subsection{Direct strain energy harvesting}

The power output that is generated by a piezoelectric energy harvester is dependent on both material properties as well as geometry of the device. In this section the material properties which are relevant for power generation as well as the geometric aspects of the energy harvester are described.
2.1.1. Material properties.

For direct strain energy harvesting, one important material property is the energy density of the material as defined in [20]:

$$
U_{\mathrm{el}}^{\mathrm{S}}=u_{\mathrm{el}}^{\mathrm{S}} S^{2}=\frac{d_{i j}^{2}}{\varepsilon^{\mathrm{T}} s^{\mathrm{E}} s^{\mathrm{D}}} S^{2},
$$

where $U_{\mathrm{el}}^{\mathrm{S}}$ is the electrical energy density under strain load, $u_{\mathrm{el}}^{\mathrm{S}}$ is the piezoelectric energy density constant under strain load, $d_{i j}$ is the piezoelectric charge constant in the direction of the applied strain, $\varepsilon^{\mathrm{T}}$ is the dielectric constant of the material (under constant stress), $s^{\mathrm{E}}$ and $s^{\mathrm{D}}$ represent the compliance under open and short circuit conditions respectively and $S$ is the applied strain. In strain governed applications such as inside an automobile tire, the strain limit $S=S_{\max }$ dictates whether or not the material can be used for direct strain energy harvesting, while $u_{\mathrm{el}}^{\mathrm{S}}$ provides information on the conversion efficiency of the material.

The properties of piezoelectric ceramic-polymer composites can be tailored in a number of different ways. These include altering the type of ceramic and polymer matrix, varying the ceramic volume fraction or altering the connectivity scheme [19]. One way of altering the connectivity scheme is by using piezoelectric fibers oriented in the poling direction. Another is by using dielectrophoretic (DEP) processing [21]. With this technique granular piezoelectric particles are aligned along the poling axis, thus achieving significantly higher piezoelectric properties at low ceramic volume fractions [22]. In this manner a higher energy density can be obtained, but the low ceramic volume fraction also ensures that the maximum strain to failure of the material remains high enough for the application.

2.1.2. Energy harvesting devices. The most basic direct strain energy harvesting device is a piezoelectric material in parallel plate configuration, which exploits the $d_{31}$ effect in piezoelectric materials, with charge output, $Q$, being proportional to [23]:

$$
Q=\frac{d_{31} S w l}{s^{\mathrm{D}}}
$$

where $w$ is the width of the device, $l$ is the length of the device and $d_{31}$ is the piezoelectric charge constant perpendicular to the poling direction ${ }^{3}$. For this equation to be valid, it is assumed that the energy harvesting device does not influence the deformation of the host structure. This is only the case when the harvester is small compared to the host structure and the material does not have a much lower compliance than the material of host structures.

In order to exploit the high longitudinal piezoelectric constants $\left(d_{33}\right)$ of the piezoelectric materials along the transverse direction of the device, an interdigitated electrode (IDE) configuration can be used. In this configuration, the total charge output of the device equals:

$$
Q=\frac{-(n-1) d_{33} S w t}{s^{\mathrm{D}}},
$$

3 Note the effective $d_{i j}$ of the device should be taken, i.e. the clamping effect of the electrodes and/or substrate foils should be taken into account. 


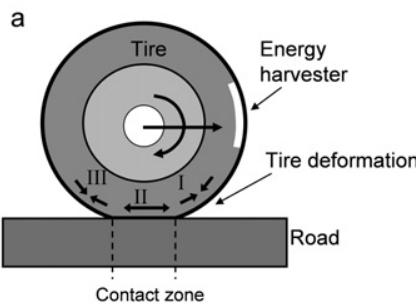

C

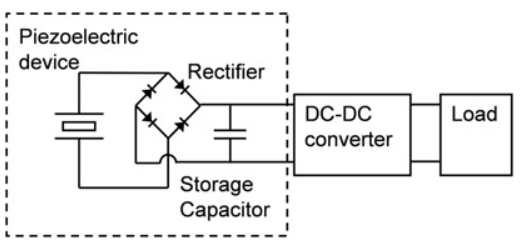

Figure 1. (a) Schematic of the deformation that an automobile tire experiences during a revolution. An energy harvesting material which is adhered to the inner liner of the tire will experience compressive stress before and after the contact zone (I and III), and tensile stress in the contact zone (II). (b) Typical charge signal that is generated by the energy harvester in a rolling tire. (c) Basic electronic circuit for one stage power management in piezoelectric based energy harvesters

where $n$ is the number electrodes in the IDE array, $d_{33}$ is effective piezoelectric charge constant of the material in poling direction and $t$ is the thickness of the device. The capacitance of an IDE device is calculated using conformal mapping techniques [24].

\subsection{Application in automobile tires}

During revolution of an automobile tire, the tread of the tire is deformed when contact is made with the road surface. As a result of this deformation, the inside surface of the inner liner of the tire on either side of the contact area will be loaded in compression, while within the contact area the inner liner of the tire is loaded in tension [25]. When a piezoelectric material is attached to the inside of the tire, the corresponding strains due to the tire-road contact are directly transferred to the energy harvester, which will generate charge as a result of this deformation (see also figure 1).

This charge is used to power an electrical load, for instance a sensor and a wireless transmitter and power management electronics are needed to adapt the power output of the energy harvester to suit the load. The power management electronics typically include a rectifier, storage capacitor and DC-DC converter to modify the current-voltage characteristics of the power source (see figure 1(c)). In this paper only the simplest energy generating system will be considered, i.e. only considering a one stage system powering the storage capacitor. Multi-stage harvesting techniques may be used to increase the harvested power [26].

Here the energy transferred to the storage capacitor, $C_{\text {store }}$, during the $n$th cycle equals:

$$
\Delta E_{n}=\frac{1}{2} C_{\text {store }}\left(V_{n}^{2}-V_{n-1}^{2}\right)
$$

where $V_{n}$ is the voltage on the storage capacitor after $n$ charging cycles. If the storage capacitor is large compared to the self-capacitance of the piezoelectric element and losses in the rectifier are neglected, this voltage can be approximated by:

$$
V_{n}=V_{n-1}+\frac{\sum_{i=1}^{4}\left(Q_{i}-\frac{2 V_{n-1}}{C_{\text {piezo }}}\right)}{C_{\text {store }}},
$$

where $Q_{i}$ is the charge generated during one quarter charge cycle (see figure 1) and $C_{\text {piezo }}$ is the capacitance of the piezoelectric element. The piezoelectric element has to be discharged and subsequently charged up to the voltage level of the storage capacitor every time the direction of the charge flow changes [27]. It is clear that the highest energy storage can be achieved by a device with a high charge output and low capacitance of the piezoelectric element. This translates to materials with a high piezoelectric charge constant and low dielectric constant, analogous to the energy density described in equation (1).

A simple two-dimensional plain strain finite element (FE) model for the strain harvesting foil attached to a rolling tire in contact with the road surface was made using COMSOL ${ }^{\circledR}$ FE package. In all FEM simulations, a triangular Lagrange-quadratic element type was used, with 2 degrees of freedom in displacement at each node and one electric potential degree of freedom. The model simulates the charge output as a function of tire displacement and can be used to evaluate the effect of several material properties such as piezoelectric properties and stiffness of the energy harvester devices. The model describes tire displacement and simulates the rolling of the tire by an applied contact force traveling along the outer surface of the tire. When the applied contact force passes the location of the energy harvester, this simulates that part of the tire touching the ground. The distribution of the applied contact force as a function of contact patch length is derived from a static FE model with contact properties. The properties of the tire in the model are thickness $t_{\text {tread }}=15 \mathrm{~mm}$ and rubber compliance $s=5 \times 10^{-8} \mathrm{~m}^{2} \mathrm{~N}^{-1}$ and Poisson's ratio $v=0.49$ for the tread and thickness $t_{\text {sidewall }}=10 \mathrm{~mm}$ and rubber compliance $s=10^{-7} \mathrm{~m}^{2} \mathrm{~N}^{-1}, v=0.49$ for the tire sidewall.

\section{Experimental details}

\subsection{Materials and device manufacturing}

The composites materials were made by applying the mixture of PU ('Crystal Clear 200' Smooth-on Inc., Easton, USA) and PZT5A4 powder (Morgan Electroceramics, Ruabon, UK) in a volume ratio of 9:1 on a gold electroded polyethylene terephthalate, (PET) film substrate. A layer thickness of $300 \mu \mathrm{m}$ was applied using a doctor blade method. The dimensions of the active area of the IDE is $40 \times 16.5 \mathrm{~mm}^{2}$, with a width and spacing of $500 \mu \mathrm{m}$ of the electrode fingers. Three different types of composites were manufactured. The first composite type (composite PC10) contained uniformly dispersed granular PZT particles. The second composite type 
Table 1. Material properties of several different composites and reference materials.

\begin{tabular}{|c|c|c|c|c|c|c|}
\hline Materials & Type & PZT volume fraction & $\varepsilon_{33} / \varepsilon_{0}(f=20 \mathrm{~Hz})(-)$ & Effective $d_{i j}{ }^{\mathrm{a}}\left(\mathrm{pC} \mathrm{N}^{-1}\right)$ & $s^{\mathrm{E}}\left(\mathrm{m}^{2} \mathrm{~N}^{-1}\right)$ & Effective $u_{\mathrm{el}}^{\mathrm{S}}\left(\mathrm{J} \mathrm{m}^{-3}\right)$ \\
\hline PC10 & $d_{33}$ & 0.10 & 7.9 & 0.18 & $8.0 \times 10^{-10}$ & $7.2 \times 10^{2}$ \\
\hline SPC10 & $d_{33}$ & 0.10 & 14 & 1.1 & $7.5 \times 10^{-10}$ & $1.8 \times 10^{4}$ \\
\hline MFC & $d_{33}$ & 0.90 & 435 & 105 & $3.3 \times 10^{-11}$ & $2.9 \times 10^{9}$ \\
\hline PVDF & $d_{31}$ & N/A & 11 & 26 & $5.0 \times 10^{-10}$ & $2.7 \times 10^{7}$ \\
\hline PZT 5A4 & $d_{31}$ & 1.0 & 1830 & 240 & $1.6 \times 10^{-11}$ & $1.8 \times 10^{10}$ \\
\hline
\end{tabular}

a The subscripts $i$ and $j$ are equal to the device type (column 2).

also contained granular PZT particles which were structured to form fibrils by the DEP process (composite SPC10) and the third composite type contained short PZT fibers structured by the DEP process (composite SFC10). The DEP settings that were used during curing of the polyurethane were: frequency $f=100 \mathrm{~Hz}$, electric field strength of $E=2 \mathrm{kV} \mathrm{mm}^{-1}$, applied for $4 \mathrm{~h}$ at room temperature and left to further cure overnight. Subsequently the composites were post cured for $4 \mathrm{~h}$ at $70^{\circ} \mathrm{C}$ and poled at $6 \mathrm{kV} \mathrm{mm}^{-1}$ (particle composites) or $4 \mathrm{kV} \mathrm{mm}^{-1}$ (fiber composites) for $30 \mathrm{~min}$ at $60^{\circ} \mathrm{C}$. The PU matrix material was chosen as a model matrix because of its convenient processing properties with regard to dielectrophoresis [28]. No attempt was made to optimize the PZT volume fraction, matrix properties or DEP settings in this work.

A macro-fiber composite (MFC, type M8528-P1, SmartMaterial Gmbh, Dresden, Germany), PVDF (type 2-10037440, Measurement Specialties Inc, Hampton, USA) and PZT 5A4 plates (Morgan Electroceramics, Ruabon, UK) with dimensions $l \times w \times t=40 \times 17 \times 0.175 \mathrm{~mm}^{3}$ were used as reference materials. The MFC and PVDF samples were also tested inside the tire as reference devices.

\subsection{Material testing}

The charge output of the materials was measured with increasing strain in a tensile test bench (500N TesT Gmbh) while the electrodes were shorted through a charge meter (Kistler 5015) and recorded on an oscilloscope (Agilent 54622A). The strain was measured by an optical dilatometer, except in case of the MFC sample where the strain was estimated from the measured stress using the linear elastic compliance [11]. Elevated temperatures measurements were performed by heating the energy harvesting device using a hot air fan while recording the temperature with a thermocouple located at the middle of the sample. Subsequently, samples were heated to a certain temperature for $3 \mathrm{~h}$ under short circuit conditions while charge output measurements at a specified strain level were performed at room temperature in between the heating steps.

\subsection{Device testing in tires}

The composites and reference materials were bonded to the inside surface of an automobile tire (Pirelli P6000 195/60 R16) using cyanoacrylate adhesive (Loctite 406) to make energy harvesting devices and their charge output measured in a rolling tire test setup. A tire was made to travel along a stiff flat track at a fixed velocity of $0.25 \mathrm{~m} \mathrm{~s}^{-1}$, by a moving axle powered by an electromotor. The surface of the flat track could be adjusted in height to increase the tire deformation. On each side the tire was attached to the setup by bolting the sidewall just under the bead wire to a stiff steel panel which was connected to the moving axle. A deflated tire was used for ease of testing and interchanging lead wires between samples. The lack of tire pressure influences the deformation of the tire. However, the principle of deformation remains the same and as all materials were measured on the same tire, a valid comparison between the materials can be made. The charge output from the devices was recorded using a charge meter (Kistler 5015) connected to an oscilloscope (Agilent 54622A). Contact length measurements were made by sliding $100 \mu \mathrm{m}$ thick foils into contact with the tire on each side and measuring the distance between them. Elevated temperature charge measurements were made by heating the tire from the outside using a hot air fan and recording the temperature on the inner surface of the device being measured. Additionally, power output measurements were performed by recording the voltage developed on a storage capacitor as a function of the number of cycles using an electrometer (Keithley 617).

\section{Results}

\subsection{Material characterization}

Micrographs of the piezoelectric composite materials manufactured in this study are presented in figure 2, revealing a successful alignment of the particles and fibers in the two structured composites as a result of the DEP structuring process. In figure 2(b) it can be seen that the DEP structuring process causes a concentration of PZT in between the electrodes.

Materials properties of the composite and reference materials are summarized in table 1 . The (small signal) energy density constant, $u_{\mathrm{el}}^{\mathrm{S}}$, of the materials is calculated using equation (1).

The average piezoelectric constants of the materials as a function of strain amplitude are presented in figure 3 . The average value of $d_{i j}$ for a certain strain amplitude, $S_{\text {peak }}$, is calculated using equations (2) and (3) using $Q=Q_{\max }$ and $S=S_{\text {peak }}$. The $d_{33}$ of the MFC material increases up to $S=0.22 \%$ after which a rapid decrease is witnessed, which did not recover when the strain amplitude was lowered. No 

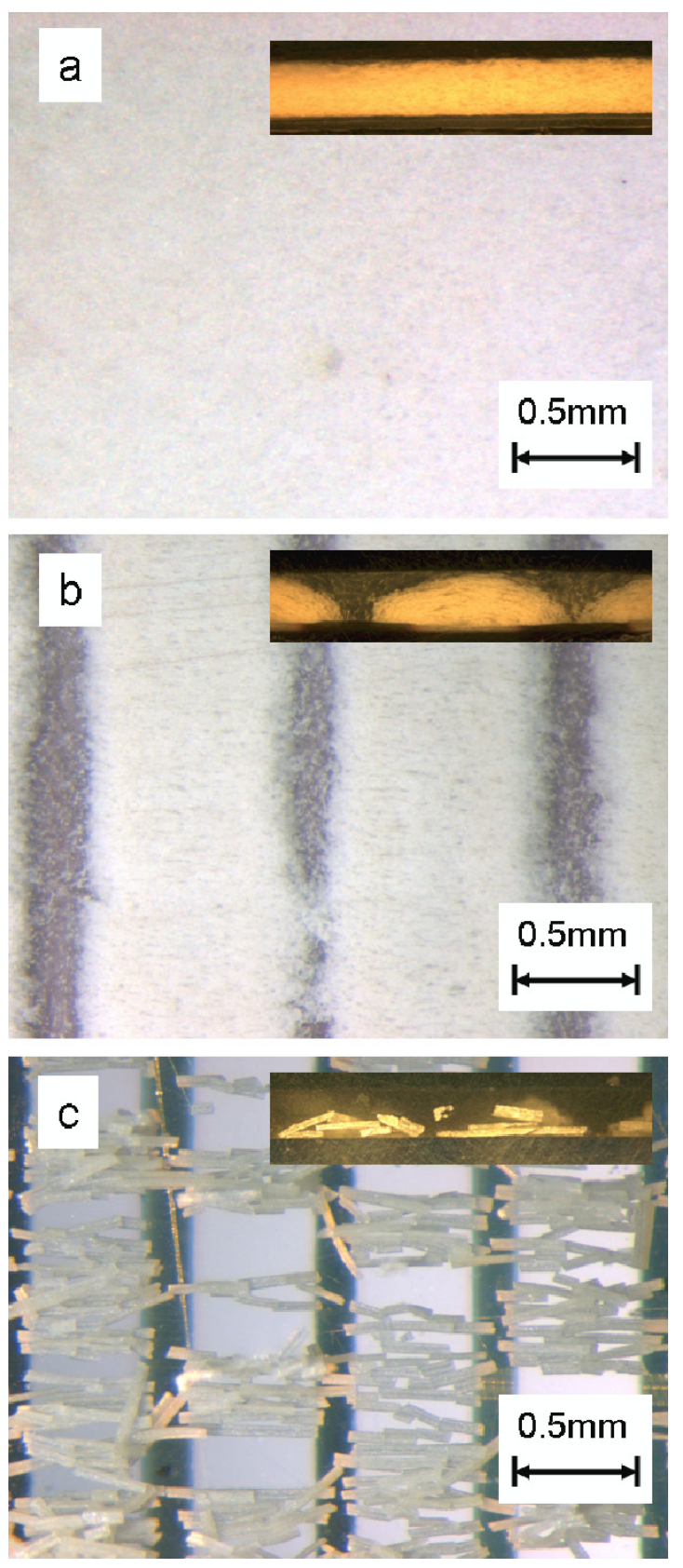

Figure 2. Micrographs of composites manufactured in this work: (a) top view of the PC10 composite (b) top view of the SPC10 composite showing oriented PZT particle chains (light) between the electrodes (dark) and (c) top view of the SFC10 composite showing PZT short fibers (light) oriented between gold electrodes (dark). In the insets are cross sections of the materials.

other samples experienced such drastic irreversible behavior in the strain region tested. Moreover, during loading and unloading cycles, the MFC material displayed a significant time hysteresis, being slow to fully discharge after higher applied strain amplitudes. The PVDF sample exhibited a slight increase in $d_{31}$ in the region tested and no significant time hysteresis was observed. The $d_{33}$ of the composite materials SFC10 and SPC10 initially increased, followed by gradual decrease at higher strains and again no significant

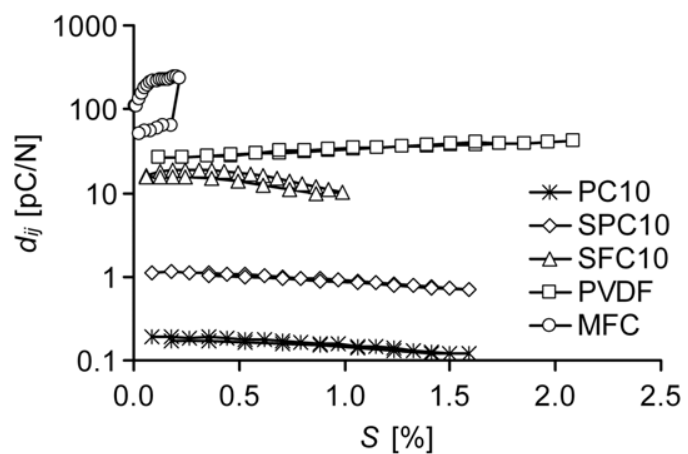

Figure 3. Piezoelectric constants $d_{i j}$ as a function of strain in the material for PC10, SPC10, SFC10, PVDF and MFC samples.
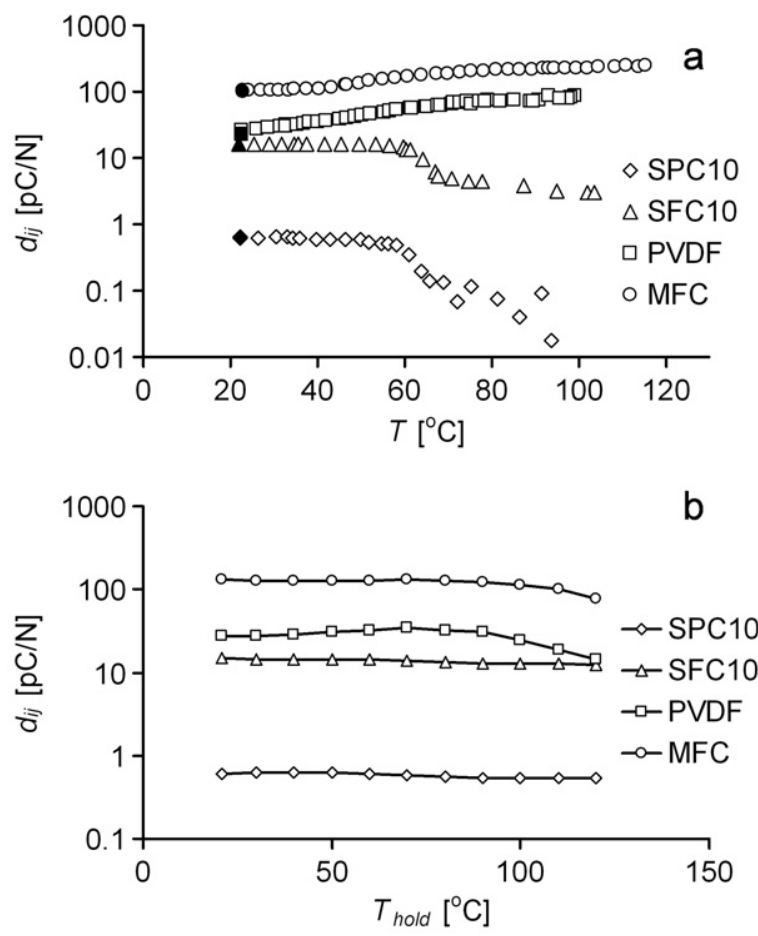

Figure 4. Piezoelectric charge constants of several materials as a function of temperature (a) and after holding for $3 \mathrm{~h}$ at elevated temperature (b). The average $d_{33}$ was measured at a strain of $S=0.3 \%$ for the SPC10 and SFC10 samples and at $S=0.06 \%$ for the MFC sample. For the PVDF sample, the $d_{31}$ was measured at $S=1 \%$. The black data points in (a) represent the measured $d_{33}$ after cooling back to room temperature.

hysteresis was observed. In the PC10 material the initial increase in $d_{33}$ was less apparent.

In figure 4(a) the average piezoelectric constants of the materials as a function of temperature are presented. The $d_{33}$ of samples SFC10 and SPC10 is relatively constant from room temperature up to $60^{\circ} \mathrm{C}$, after which a rapid decrease is observed. However, upon cooling the $d_{33}$ is restored. The MFC and PVDF reference samples displayed opposite behavior: an initial increase with increasing temperature, but after cooling a slight decrease in room temperature values are observed. In figure 4(b) the influence of holding the samples at elevated temperatures on the room temperature piezoelectric 


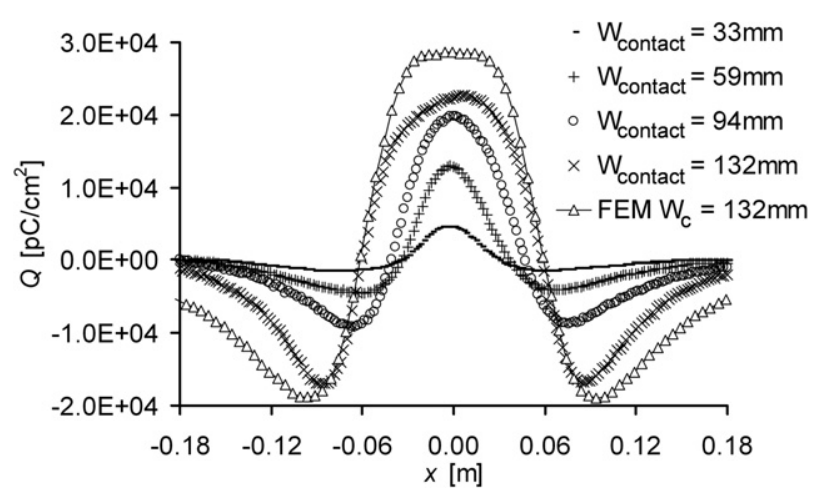

Figure 5. Typical charge profiles for a piezoelectric composite energy harvester (sample SFC10): area normalized charge output $Q$ as a function of travel distance of the tire for several tire-road contact patch lengths. At $W_{\text {contact }}=132 \mathrm{~mm}$, the simulated charge output using the FEM model is presented for comparison.

constants of the materials after cooling back down to room temperature is presented. The room temperature piezoelectric constants of the MFC and PVDF samples decrease rapidly with increasing holding temperature above about $90^{\circ} \mathrm{C}$, whereas the composite SFC10 sample does not.

\subsection{Output of the devices in the tire}

The charge output of the sample as a function of travel distance of the tire is presented in figure 5. An increase in tire contact length (a higher load on the tire) results in a higher maximum charge output for the device.

The peak-to-peak charge output of the different devices as a function of tire-road contact length (see figure 6(a)) shows an increase in charge output with increasing contact length. At a large contact length, the influence of high strain on the output of the devices is plotted (see figure 6(b)). The fiber and particle composites as well as the PVDF device do not show any degradation in output signal in the first 100 cycles, but the signal of the MFC composites decays. The influence of temperature on the output of the devices is presented in figure 7. The output of SFC10 and SPC10 composite materials decreases at temperature higher than $50^{\circ} \mathrm{C}$, however the charge output recovers to its initial value when the tire is cooled down to room temperature. The PVDF and MFC devices initially display an increase in charge output as a function of increasing temperature. However, the output of both PVDF and MFC samples starts to reduce at $60^{\circ} \mathrm{C}$. This reduction in charge output is not recovered when the tire is cooled back down to room temperature.

The voltage measured on the storage capacitors of several different energy harvesting devices as a function of number of tire revolutions is presented in figure 8 along with the calculated added energy per cycle based on the measured storage capacitor voltage (equation (5)) and the voltage and corresponding calculated energy output based on the closed circuit charge output (equation (4)). The values of the storage capacitors were chosen such that the maximum electrical energy generated was achieved in a relatively short number of cycles. The magnitude of the energy transfer does not depend
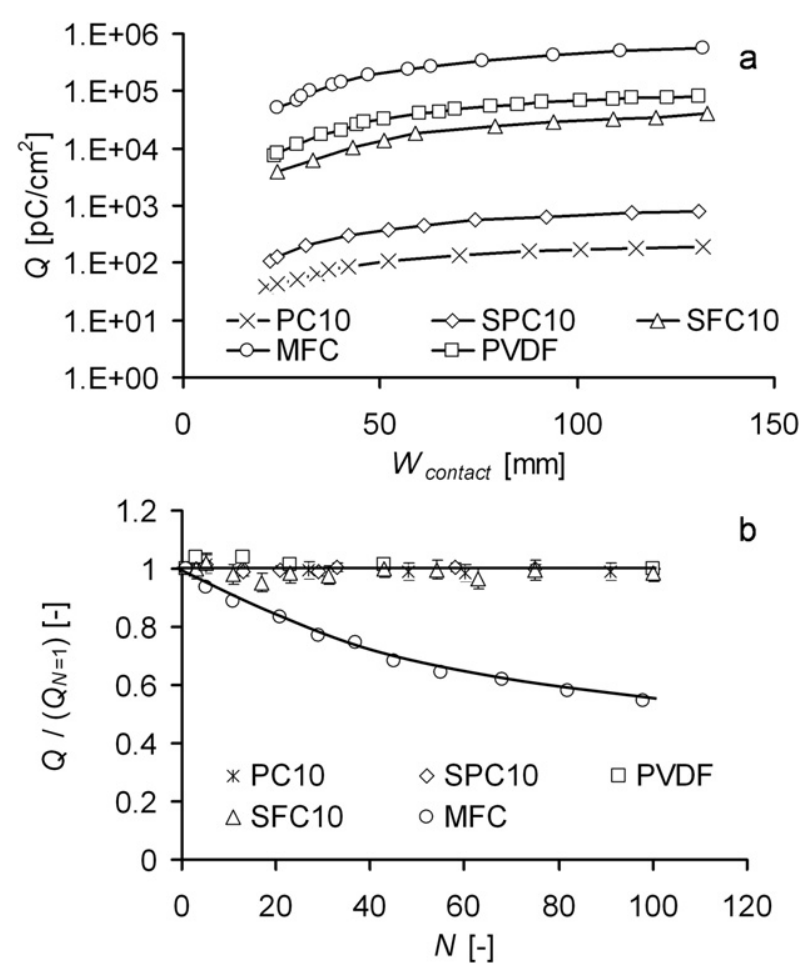

Figure 6. (a) Charge output per unit area, $Q$, of the composite and reference devices as a function of tire-road contact patch length, $W_{\text {contact }}$ (b) Charge output per unit area of several devices as a function of number of cycles at a contact length of $W_{\text {contact }}=130 \mathrm{~mm}$.

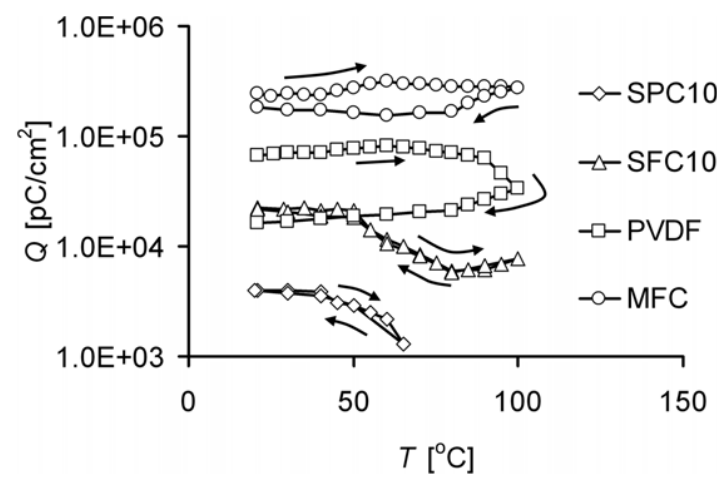

Figure 7. Charge output per unit area as a function of tire temperature for contact length of $W_{\text {contact }}=90 \mathrm{~mm}$.

on the value of the storage capacitor, it merely influences the charging time. This experiment was performed after the elevated temperature experiments (results figure 7), therefore the MFC and PVDF samples were already degraded.

\section{Discussion}

The results in figure 2 depict the effect that the DEP processing has on the microstructure of the composite materials. Both the PZT particle and PZT fibers are aligned in the direction perpendicular to the electrodes. The DEP process increases the piezoelectric constants of the composites in the poling direction and in the IDE devices also locally 

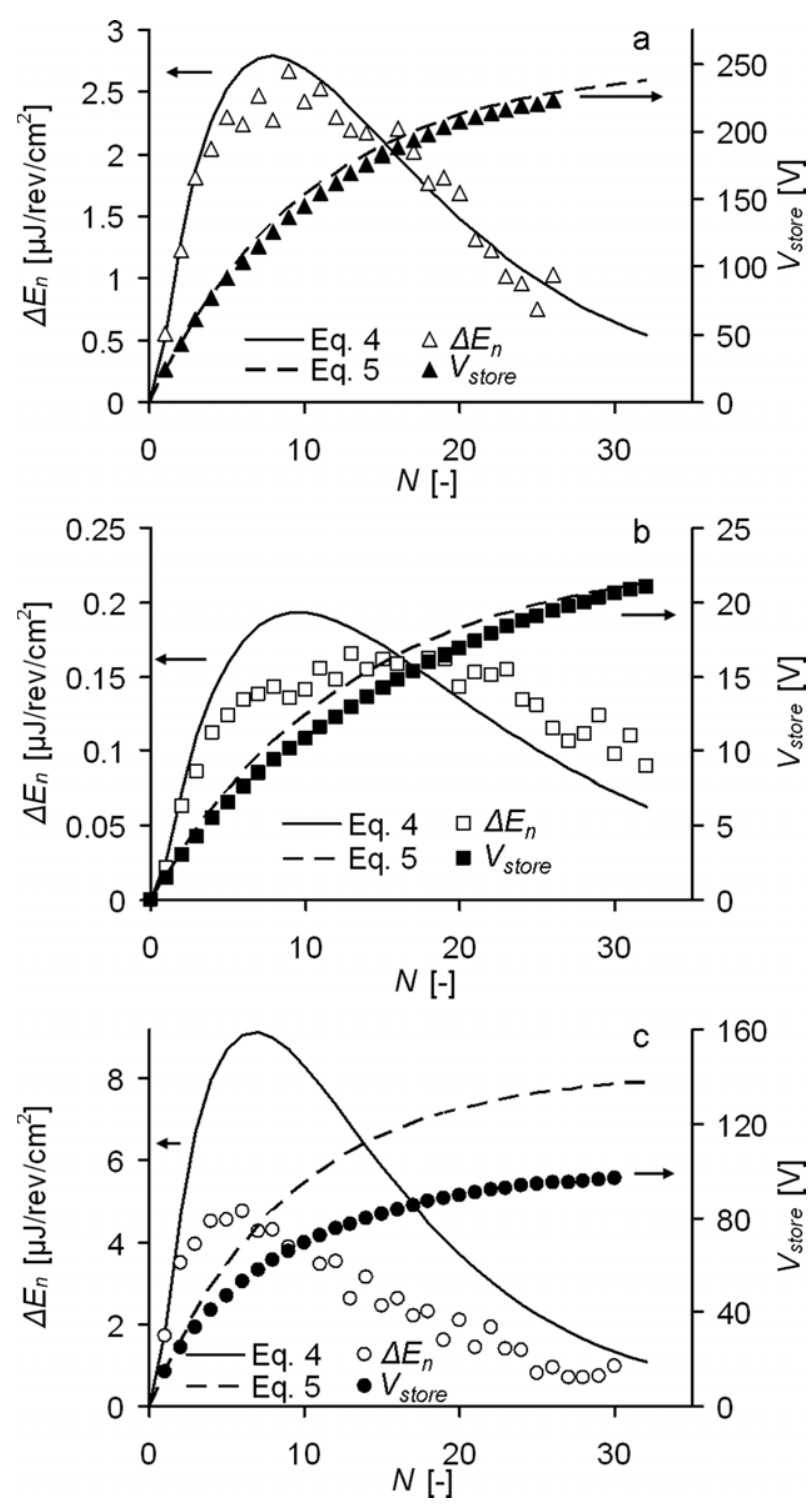

Figure 8. Power output per unit area, $Q$, of the composite and reference devices as a function of tire revolutions for samples (a) SFC10 tire-road contact patch length, $W_{\text {contact }}=100 \mathrm{~mm}$, $C_{\text {store }}=12.5 \mathrm{nF}$. (b) PVDF $W_{\text {contact }}=100 \mathrm{~mm}, C_{\text {store }}=134 \mathrm{nF}$. (c) $\mathrm{MFC} W_{\text {contact }}=100 \mathrm{~mm}, C_{\text {store }}=390 \mathrm{nF}$.

increases the PZT volume fraction in the region between the electrodes [29], see also the inset of figure 2(c). At $10 \%$ PZT volume fraction bulk values of $d_{33}$ are approximately five times higher for structured composites compared to 0-3 composites [22]. The ratio in calculated $d_{33}$ between the unstructured (PC10) and structured samples (SPC10) is 6.2, which is slightly higher than the bulk ratio. This increase is attributed to the higher concentration of PZT between the electrodes due to the DEP process. Both absolute $d_{33}$ values are significantly lower than would be expected for $0-3$ composites with a volume fraction of $10 \%$ PZT. This has two reasons: the clamping effect of the PET substrate is relatively large, as its thickness and stiffness are comparable to that of the composite and the electric field is not uniformly aligned in the same direction as the strain. In the inset of figure 2(b), the cross section of the SPC10 material reveals an arched structure of the aligned particles between the electrodes. This is the result of a mismatch between the direction of electric field during alignment and the applied strain during operation, leading to a low effective structuring factor, $\overline{P_{2}}$ [22], with respect to the applied strain direction. The $d_{33}$ increases with the structuring factor and it is anticipated that the enhancing effect of the DEP structuring may be improved if the layer thickness of the piezoelectric composite layers is decreased. Especially in the case of the short fiber composite, a single layer of evenly spaced, well oriented fibers will yield a significant improvement in $d_{33}$ with respect to the fibers in SFC10 sample which were found to be clustered and sometimes formed multiple layers.

From a comparison of the calculated energy density constant, $u_{\mathrm{el}}^{\mathrm{S}}$, of the materials as shown in table 1 , it can be observed that the energy density of the particle filled composites is orders of magnitude lower than the other materials. The DEP process increases the energy density of the material by almost a factor 10 . When replacing the particles with short fibers, the energy density constant of the material rapidly increases even more and becomes comparable to the order of magnitude of the PVDF. The highest strain energy density constant measured is for PZT5A4 for which $u_{\mathrm{el}}^{\mathrm{S}}=1.8 \times 10^{10} \mathrm{~J} \mathrm{~m}^{-3}$. This value is slightly higher than the literature value of $u_{\mathrm{el}}^{\mathrm{S}}$ reported for PZT5A [20]. This difference is caused by the differences in piezoelectric material grade and the fact that piezoelectric charge constants are highly frequency dependent, generally being higher at low frequencies [30].

The piezoelectric constants of all materials initially increase with the applied strain (figure 3 ). In the PZT-polymer composites a small increase of $d_{33}$ is witnessed after followed by a decrease. However, this decrease is limited and upon reducing the applied strain amplitude back to zero the initial values are largely recovered. No cracks were observed and the decrease in measured $d_{33}$ may be the result of limited depolarization or locally high deformations in the matrix, which reduces electrical connectivity and mechanical loading of the particles. These explanations are supported by the fact that the $d_{33}$ of the particle composite sample SPC10, starts to decrease at a much lower strain and temperature than the short fiber based composite SFC10, but is more resistant to depolarization. The PVDF sample exhibits this increase in the full range tested. It is well known that the piezoelectric effect of PVDF polymers is retained up to high strains [18]. The piezoelectric constant of the MFC composite increases with applied strain up to a maximum at $S=0.22 \%$ after which a large decrease was witnessed. The increase of $d_{33}$ is large at low applied strain, but from $S=0.08 \%$ the slope starts to level off, which is expected to be the result of depolarization of the PZT material [11]. The drop at $S=0.22 \%$ is not recovered when reducing the applied load and upon inspection cracks in the MFC were witnessed running perpendicular to the loading direction, originating from the PZT phase. After cracking of the PZT, the load must be carried by the matrix in these regions and local crazing of the epoxy matrix in the region around the cracks occurs. This locally alters the strain state 
in the material and reduces the total strain experienced by the PZT phase with respect to the total strain acting on the composite. This reduces the total charge output and hence the measured $d_{33}$.

The change in $d_{i j}$ constants with increasing temperature is governed by the connectivity of the piezoelectric phases in combination with the matrix properties. In case of the piezoelectric composites SPC10 and SFC10, the piezoelectric phase is not fully connected in the direction of the applied strain. Therefore the $d_{33}$ of these materials is governed by the matrix properties at higher temperature. The glass transition temperature $\left(T_{\mathrm{g}}\right)$ of the PU matrix is clearly visible at around $60^{\circ} \mathrm{C}$ (figure $4(\mathrm{a})$ ). In the PVDF and MFC devices the piezoelectric phase is fully connected and the temperature dependence of the $d_{i j}$ constants dominates. These are known to increase with temperature $[31,32]$.

The decrease in room temperature $d_{i j}$ constants due to thermal degradation at elevated temperatures is most notable for the reference samples (figure 4(b)). The changes in piezoelectric constant measured in the SFC10 sample are small and may be the result of high temperature relaxations in the material. The PVDF material is well known for its poor temperature stability [32]. The degradation of the MFC above $90^{\circ} \mathrm{C}$ is also significant. It must be noted that the temperature dependence of the MFC was measured on a sample which was already partially mechanically depoled and had several cracks. It is probable that relaxations in the material at high temperatures could lead to local changes in connectivity after cooling, which can have severe influences on the piezoelectric constants.

From the charge output profile of the devices mounted in the tire (figure 5), it can bee seen that an increase in contact length leads to an increase in tire strain (both compressive and tensile components) and therefore an increase in charge output. No significant influence of rubber hysteresis can be witnessed in the individual charge output profiles. This is probably due to the clamping effect of the energy harvesters. However, a significant influence of relaxation (attributed to the tire rubber) was observed when tire was stopped while the device was in the middle of the contact patch. The effect was different for the different materials. The charge of the SPC10, SFC10 and PVDF materials was seen to follow the relaxation of the tire strain. However, the MFC output remained relatively stable after stopping the tire. This is due to strong retardation effects in the soft PZT [31]. Due to the difference in connectivity of the PZT phase between the SPC10 and SFC10 samples and the MFC material, the PZT in the MFC composite is being strained up to much higher levels which gives rise to much higher hysteresis levels [30, 33].

The peak-to-peak output of all devices increases with tire-road contact length, which is related to an increase in strain in the inner liner due to the larger deformations in the tire (figure 6(a)). The SFC10 sample exhibits a gradual decrease in charge output up to about $50{ }^{\circ} \mathrm{C}$ after which a sudden drop occurs. This is about $10^{\circ} \mathrm{C}$ lower than the onset of the decrease in $d_{33}$ in the material itself (see also figure 4(a)) and is again related to the $T_{\mathrm{g}}$ of the PU matrix used in this composite. Below the $T_{\mathrm{g}}$ of the polymer, a relatively constant output is observed. The temperature at which the decrease in power output occurs can easily be controlled be changing the matrix material to a higher $T_{\mathrm{g}}$ matrix. Moreover, this type of behavior can also be used as a tire temperature sensor to warn against overheating, which is a significant cause of tire failure [34, 35]. At a fixed contact length, devices with higher piezoelectric constants and stiffness lead to a higher charge output per unit area. This also follows from equations (2) and (3). However, the strains experienced during loading cause depolarization and therefore a loss of charge output for the MFC device (figure 6(b)). This effect is magnified at higher temperatures (figure 7). Upon inspection after the temperature loading experiments several cracks were found similar to cracks found in the tensile strained MFC sample.

For the PVDF the loss in charge output of the device in the tire is much greater than the reduction in material properties would predict. This may be caused by partial debonding of one of the electrodes during the combination of loading at high temperatures. Based on the charge output of the energy harvesting devices, an estimate of the strain in the inner liner of the tire can be made using equations (2) and (3). From the peak measured charge at a contact length of $W_{\mathrm{c}}=100 \mathrm{~mm}$ of the SPC10 composite an estimated peak strain $S=0.49 \%$ is calculated and for SFC10 a maximum estimated strain of $0.48 \%$ occurs. From the charge output of the PVDF reference sample, a maximum estimated strain of $S=0.69 \%$ is present, though for the MFC reference sample a mere estimated strain of is $S=0.18 \%$ predicted. The predicted strain by the PVDF sample is slightly higher than the estimated strain in the SPC10 and SFC10 composite samples. The reason for this difference in estimated values is the stiffness mismatch between the harvesting device and tire rubber. In the case of the SPC10 and SFC10 samples, the substrate influence also plays a part. The energy harvesting materials themselves influence the measured strain, because the materials are in general stiffer than the rubber of the inner liner [36]. Obviously the clamping effect is lowest for the thin, compliant PVDF sample and much higher in the relatively thick and stiff MFC device.

The strain in the center of the inner liner of a tire during operation varies with inflation pressure and loading force which both influence the contact patch length. Typical maximum compressive and tensile strains experienced in the tire inner liner during one revolution are reported to be around $-0.28 \%<S<0.72 \%$ for a standard automobile tire at a load of $600 \mathrm{~kg}$. This corresponds to a contact length of approximately $115 \mathrm{~mm}$ [14]. This strain was measured using a PVDF strain gage and is subject to the same discussion as presented in the previous paragraph. Its value is similar to the our result with the deflated tire at a contact length of $W_{\mathrm{c}}=100 \mathrm{~mm}$. The strain varies significantly at other locations in the tire cross section [37]. Moreover, the tensile and compressive strains can easily peak at higher values, for instance during braking [39] or driving over rough terrain. During normal operation the temperature on the inner liner of an automobile tire can rise to $100^{\circ} \mathrm{C}$ depending on inflation pressure, loading force, speed and 

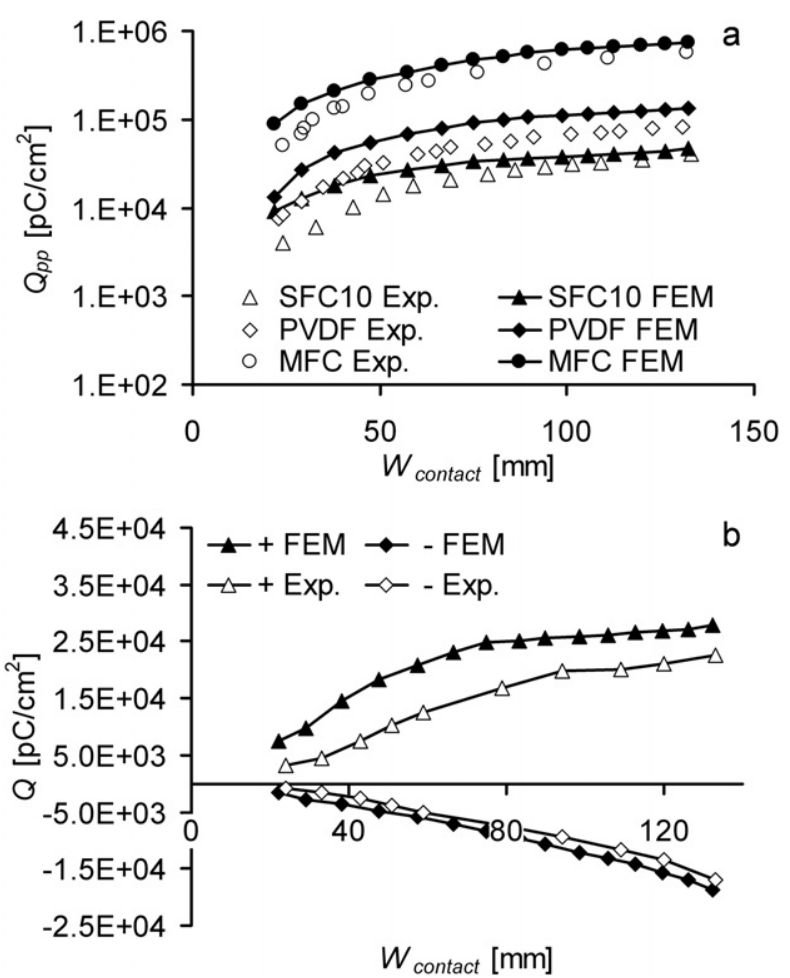

Figure 9. (a) Experimental results and FEM calculations for the area normalized charge output of the SFC10, PVDF and MFC devices as a function of $W_{\text {contact }}$. (b) A comparison of experimental and FEM results of charge values corresponding the tensile $(+)$ and compression (-) peaks during loading of SFC10 in the tire (see figure 1 for a description of tensile and compression regions in the tire).

ambient temperature [38, 34]. For (light) truck tires where self-heating is more dominant, even higher temperatures may be reached [35].

Evidently, based on these typical temperatures and strains in a typical tire operating environment, the concept of direct strain energy harvesting is difficult to accomplish using state-of-the-art piezoelectric materials. However, contrary to the fixed material properties of PZT and PVDF materials the composite material properties can potentially be modified to withstand the tire operating conditions if a more temperature stable matrix is used. Presently, even using the low- $T_{\mathrm{g}} \mathrm{PU}$ matrix, the room temperature properties are largely retained after heating even though the output diminishes at high temperature.

The normalized charge output results from FEM analysis compare reasonably well with the experimental values, given the simplifying assumptions made in the model. With increasing tire-road contact length the ratio of compressive and tensile contributions to the measured charge output change, a trend which is also seen in the FEM results. This indicates that the calculated deformation in the inner liner as a function of contact length is realistic. The slope of the tensile strain increase changes at a contact length of around $W_{\mathrm{c}}=$ 80-100 mm, which is comparable to the experimental results. However, the FEM simulations systematically overestimate the charge output of the devices, which can be attributed to

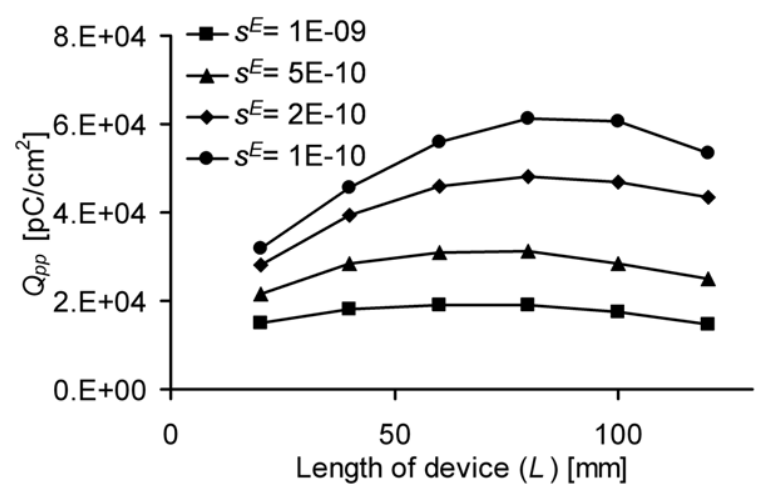

Figure 10. FEM calculations for the area normalized charge output of energy harvesting devices with different compliances as a function of device length for a fixed contact length of $100 \mathrm{~mm}$.

a number of simplifications in the FEM model. The FEM model is two-dimensional and does not take into account the composite structure of the tire, the losses due to an adhesive layer and finally the nonlinearity of the materials properties. In actual automobile tires the highest deformations occur in the outer tread because of the presence of stiff reinforced rubber composite belt materials in the inner part of the tire tread [34]. This difference will be strongest in the part of the tire in contact with the ground, which correlates to the results in figure 9(b), where especially the charge output of the tensile region is overestimated.

Based on the fact that the FEM model predicts the same trends as the experiments, it can be used to design improved direct strain energy harvesting devices taking into account counteracting dependences. In figure 10 the effect of foil stiffness on the maximum charge output as a function of the device length is shown.

Firstly, the increase in device stiffness causes a higher charge output in strain governed loading (see equations (2) and (3)), but a higher stiffness also causes a higher clamping effect. This clamping effect is greatest at the edges of the device. In the region around the device the tire rubber is free to deform and therefore the greatest influence of this negative effect is for short devices, as can be seen in figure 10 .

The calculated energy output of the devices based on charge output of the piezoelectric materials corresponds well to the energy output calculated from the voltage measured on the storage capacitor (see figure 8), especially in the case of the SFC10 material. Moreover the normalized charge output of the SFC10 material is higher than the degraded PVDF and comparable to the degraded MFC sample.

In case of the MFC device the output is different than the predicted results using equation (4). The lower energy output profile is partially the result of further degradation of the material during consecutive runs. However, the discrepancy is much higher than would be expected based on material degradation alone, as the MFC sample was already partially degraded during the measurement and the output was therefore relatively stable. The MFC material showed a significant amount of time hysteresis between loading and unloading, which is not uncommon for a soft PZT [30]. The compressive stress is generally lower than the maximum 
Table 2. Estimated power output of different devices at a speed of $50 \mathrm{~km} \mathrm{~h}^{-1}$ based on maximum energy per revolution cycle and the corresponding area needed for a power output of $P=100 \mu \mathrm{W}$. For the PVDF and MFC reference devices the initial and degraded values are presented.

\begin{tabular}{llll}
\hline Device & $\begin{array}{l}\Delta E_{n, \max } \\
\left(\mu \mathrm{J} \mathrm{rev}^{-1} \mathrm{~cm}^{-2}\right)\end{array}$ & $\begin{array}{l}P_{\max } 50 \mathrm{~km} \mathrm{~h}^{-1} \\
\left(\mu \mathrm{W} \mathrm{cm}^{-2}\right)\end{array}$ & $\begin{array}{l}\left.A\right|_{P=100 \mu \mathrm{W}} \\
\left(\mathrm{cm}^{2}\right)\end{array}$ \\
\hline PC10 & 0.00054 & 0.0096 & 10440 \\
SPC10 & 0.013 & 0.19 & 522 \\
SFC10 & 2.7 & 30 & 3.3 \\
PVDF (init.) & 5.2 & 40 & 2.5 \\
PVDF (dgr.) & 0.17 & 1.3 & 80 \\
MFC (init.) & 54 & 399 & 0.25 \\
MFC (dgr.) & 4.8 & 35 & 2.8 \\
\hline
\end{tabular}

tensile stress during one revolution. This causes hysteresis in the charge output of the PZT and thus a reduction in the charge transferred to the storage capacitor. Moreover, the device undergoes two consecutive compressive cycles during repetitive revolutions, which increases the hysteresis effects. For a cumulative hysteresis of about $23 \%$ the calculated energy output as a function of number of revolutions matches the experimental values.

The SFC10 sample did not show such significant hysteresis behavior and hence the calculated energy output corresponds well to the measured output and the generated energy per unit area is comparable to the MFC sample. However, the SFC10 composite material delivers its peak energy output at a fairly high voltage (approximately $120 \mathrm{~V}$ ). In practice, $V_{\max }$ may be limited by power conditioning electronics, as step down voltage converters are limited in their range. However, high voltage storage devices with efficiencies of almost $100 \%$ have been demonstrated for single stage power harvesting [26]. Therefore, if higher voltages could be utilized in the power storage device the direct strain SFC10 type materials would be an interesting power source.

Based on the charge output of the devices the maximum power output during operating conditions in a tire can be estimated. The difference in stiffness between the energy harvester and the tire is strain rate dependent; this may affect strain transfer in dynamic operation, probably increasing the power output at high speeds. However, the composite matrices and PVDF are also viscoelastic, which will counteract the effect of viscoelasticity of the rubber. Moreover, the $d_{33}$ of the PZT is also strain rate dependent which will also lead to lower relative output at higher speed [30]. Without the influence of strain rate dependence, the predicted power output is presented in table 2 . In all cases, the capacitance of the rectifier is assumed much smaller than the capacitance of the energy harvesting device.

At present approximately $14 \%$ of the automobile tire inner surface would be covered with SPC10 material if $100 \mu \mathrm{W}$ output is desired while traveling at $50 \mathrm{~km} \mathrm{~h}^{-1}$, which is sufficient for simple measurements such as temperature or pressure monitoring at intermediate intervals [2]. However, using only low grade materials, this material is potentially very cost effective to manufacture. Room for improving the power output is available by increasing the efficiency of the microstructure. Moreover, when fibers are used as filler material instead of particles, a mere $0.1 \%$ of the automobile tire is covered $\left(3.3 \mathrm{~cm}^{2}\right)$ leaving plenty of room for increase of the power for extra sensing and transmitting purposes.

\section{Conclusions}

Low cost foil based materials for direct strain energy harvesting in automobile tires were developed using piezoelectric PZT-polymer composites. At present, the output of the composite samples is lower than virgin state-of-the-art piezoelectric materials such as MFC composites and PVDF polymer, but both MFC (strain) and PVDF (temperature) were shown to degrade significantly in environments comparable to operating environments in automobile tires. The room temperature power output of fiber based composites is higher than PVDF sample and almost comparable to MFC sample after degradation. An analytical model was developed relating the charge output profile of the energy harvester to the power transferred to a storage capacitor.

Analytical results correspond reasonably well to experimental results for power output of several devices, except for the MFC device where a significantly lower experimental output is measured. This is probably due to hysteresis in the PZT ceramic. Results from a simple 2D FEM model correspond reasonably well with measured charge output of actual devices. At present dielectrophoretically processed short fiber composites provide an estimated power of $30 \mu \mathrm{W} \mathrm{cm}{ }^{-2}$ at modest traveling speeds of $50 \mathrm{~km} \mathrm{~h}^{-1}$. If the power output can be increased by further materials development, the piezoelectric polymer-PZT composite materials manufactured in this study are to be considered as serious candidates for direct strain energy harvesting from tire deformations.

\section{Acknowledgments}

This work was financially supported by the Smartmix funding program (grant SMVA0607), as part of the program 'Smart systems based on integrated Piezo'. The authors are grateful to Morgan Electro Ceramics (Ruabon, United Kingdom) for providing the PZT used in this study.

\section{References}

[1] Park G, Rosing T, Todd M D, Farrar C R and Hodgkiss W 2008 J. Infrastruct. Syst. 14 64-79

[2] Vullers R J M, van Schaijk R, Doms I, Van Hoof C and Mertens R 2009 Solid State Electron. 53 684-93

[3] Anton S R, Erturk A and Inman D J 2010 Smart Mater. Struct. 19115021

[4] Roundy S J, Wright P K and Rabaey J 2003 Comput. Commun. 26 1131-44

[5] Cook-Chennault K A, Thambi N and Sastry A M 2008 Smart Mater. Struct. 17043001

[6] Arms S W, Townsend C P, Churchill D L, Moon S M and Phan N 2006 HUMS: Proc. Health and Usage Monitoring Systems Conf. (Phoenix, AZ)

[7] Lang S B and Muensit S 2006 Appl. Phys. A 85 125-34 
[8] Beeby S P, Tudor M J and White N M 2006 Meas. Sci. Technol. 17 R175-95

[9] Roundy S J 2003 Energy harvesting for wireless sensor nodes with a focus on vibration to electricity conversion $P h D$ Thesis University of California, Berkeley, chapter 6

[10] Roundy S J and Wright P K 2004 Smart Mater. Struct. 13 1131-42

[11] Daue T P, Kunzmann J and Schönecker A 2008 Energy harvesting using piezo-electric macro fibre composites Smart Material Technical Publication www.smart-material.com (accessed 04-2011)

[12] Matsuzaki R and Todoroki A 2008 Sensors 8 8123-38

[13] Hatipoglu G and Ürey H 2010 Smart Mater. Struct. 19015022

[14] Apollo Project Final Report 2005 Intelligent Tire for Accident-Free Traffic IST-2001-34372

[15] Ledenbach G W 1980 A piezo powered Ic transmitter IEEE Trans. Consum. Electron. 26 229-33

[16] Moon K, Liang H, Yi J and Mika B 2007 Proc. SPIE $\mathbf{6 5 2 9} 65290 \mathrm{~K}$

[17] van den Ende D A, Bos B and Groen W A 2009 J. Electroceram. 22 185-91

[18] Wang Y, Ren K and Zhang Q M 2007 Appl. Phys. Lett. 91222905

[19] Newnham R E, Skinner D P and Cross L E 1978 Mater. Res. Bull. 13525

[20] Rödig T and Schönecker A 2010 J. Am. Ceram. Soc. 93 901-12

[21] Bowen C P, Newnham R E and Randall C A 1998 J. Mater. Res. 13 205-10

[22] van den Ende D A, Bory B F, Groen W A and van der Zwaag S 2010 J. Appl. Phys. 107024107

[23] Germano C P 1999 Morgan Electroceramics Technical Publication TP-222, Piezoelectricity www.morganelectroceramics.com (accessed 03-2011)
[24] Igreja R and Dias C J 2004 Sensors Actuators A 112 291-301

[25] Matsuzaki R and Todoroki A 2005 Sensors Actuators A $119323-31$

[26] Guan M J and Liao W H 2007 Smart Mater. Struct. 16 498-505

[27] Shu Y C and Lien I C 2006 Smart Mater. Struct. 15 1499-512

[28] Bowen C P, Shrout T R, Newnham R E and Randall C A 1997 J. Mater. Res. 122345

[29] Pohl H A 1978 Dielectrophoresis: The Behavior of Neutral Matter in Nonuniform Electric Fields (Cambridge: Cambridge University Press)

[30] Damjanovic D 2006 The Science of Hysteresis vol 3, ed I Mayergoyz and G Bertotti (Amsterdam: Elsevier) p 339

[31] Hall D A 2001 J. Mater. Sci. 36 4575-601

[32] Dargaville T R, Celina M and Chaplya P M 2005 J. Polym. Sci. B 43 1310-20

[33] Moulson A J and Herbert J M 2003 Electroceramics: Materials, Properties, Applications (Hoboken, NJ: Wiley) p 365

[34] Gardner J D and Queiser B J 2006 The Pneumatic Tire ed A N Gent and J D Walter (Washington, DC: Publication of the US Department of Transportation) pp 621-4 (DOT HS 810 561)

[35] Sokolov S L 2009 J. Mach. Manuf. Reliab. 38 310-4

[36] Matsuzaki R, Keating T, Todoroki A and Hiraoka N 2008 Sensors Actuators A 148 1-9

[37] Hall W, Mottram J T and Jones R P 2004 Proc. Inst. Mech. Eng. D 2181393

[38] Lin Y J and Hwang S J 2004 Math. Comput. Simul. 67 235-49

[39] Matsuzaki R and Todoroki A 2008 J. Sol. Mech. Mater. Eng. 2 269-80 